\title{
Short-Term Electricity Demand Forecasting Using Double Seasonal Exponential Smoothing
}

\author{
James W. Taylor \\ Saïd Business School \\ University of Oxford
}

Journal of Operational Research Society, 2003, Vol. 54, pp. 799-805.

Address for Correspondence:

James W. Taylor

Saïd Business School

University of Oxford

Park End Street

Oxford OX1 1HP, UK

Tel: +44 (0)1865 288927

Fax: +44 (0)1865 288805

Email: james.taylor@sbs.ox.ac.uk 


\title{
Short-Term Electricity Demand Forecasting Using Double Seasonal Exponential Smoothing
}

\begin{abstract}
This paper considers univariate online electricity demand forecasting for lead times from a half-hour-ahead to a day-ahead. A time series of demand recorded at half-hourly intervals contains more than one seasonal pattern. A within-day seasonal cycle is apparent from the similarity of the demand profile from one day to the next, and a within-week seasonal cycle is evident when one compares the demand on the corresponding day of adjacent weeks. There is strong appeal in using a forecasting method that is able to capture both seasonalities. The multiplicative seasonal ARIMA model has been adapted for this purpose. In this paper, we adapt the Holt-Winters exponential smoothing formulation so that it can accommodate two seasonalities. We correct for residual autocorrelation using a simple autoregressive model. The forecasts produced by the new double seasonal Holt-Winters method outperform those from traditional Holt-Winters and from a well-specified multiplicative double seasonal ARIMA model.
\end{abstract}

Key words: electricity demand forecasting; Holt-Winters exponential smoothing. 


\section{Introduction}

Online electricity demand prediction is required for the control and scheduling of power systems. The forecasts are required for lead times from a minute-ahead to a day-ahead. At National Grid, which is responsible for the transmission of electricity in England and Wales, online prediction is based on half-hourly data. A profiling heuristic is used to produce forecasts for each minute by interpolating between each half-hourly prediction. The National Grid one hour-ahead forecasts are a key input to the balancing market, which operates on a rolling one hour-ahead basis to balance supply and demand after the closure of bi-lateral trading between generators and suppliers.

Weather is a key influence on the variation in electricity demand (see Taylor and Buizza $\left.^{1,2}\right)$. However, in a real-time online forecasting environment, multivariate modelling is usually considered impractical. A multivariate online system would be very demanding in terms of weather forecast input and would require default procedures in order to ensure robustness $^{3}$. Univariate methods are considered to be sufficient for the short lead times involved because the weather variables tend to change in a smooth fashion, which will be captured in the demand series itself.

In this paper, we consider online, univariate forecasting of half-hourly data. A time series of electricity demand recorded at half-hourly intervals contains more than one seasonal pattern. Figure 1 shows half-hourly demand in England and Wales for a fortnight in June 2000. A within-day seasonal cycle, of duration 48 half-hour periods, is apparent from the similarity of the demand profile from one day to the next, particularly on weekdays. A within-week seasonal cycle, of duration 336 half-hour periods, is evident when one compares the demand on the corresponding day of adjacent weeks. There is strong appeal in using a forecasting method that is able to capture information in both seasonalities. 
Holt-Winters exponential smoothing is a popular approach to forecasting seasonal time series. The robustness and accuracy of exponential smoothing methods has led to their widespread use in applications where a large number of series necessitates an automated procedure, such as inventory control. This suggests that Holt-Winters might be a reasonable candidate for the automated application of online electricity demand forecasting. However, the method is only able to accommodate one seasonal pattern. The multiplicative seasonal ARIMA model has been extended in order to model the within-day and within-week seasonalities in electricity demand. In this paper, we adapt the Holt-Winters method so that it can accommodate two seasonalities. This involves the introduction of an additional seasonal index and an extra smoothing equation for the new seasonal index.

In the next section, we describe how ARIMA models have been adapted for online electricity demand forecasting, in order to capture multiple seasonalities in the demand series. We then show how the Holt-Winters method can be adapted for series with more than one seasonality. The section that follows presents an empirical forecast comparison of the new formulation with the standard Holt-Winters method and with a multiplicative double seasonal ARIMA model. In the final section, we provide a summary and conclusion.

\section{Multiplicative Double Seasonal ARIMA Models}

The literature on short-term load forecasting contains a variety of univariate methods that could be implemented in an online prediction system. The range of different approaches includes state space methods with the Kalman filter (e.g. Infield and Hill ${ }^{4}$ ), general exponential smoothing (e.g. Christiaanse ${ }^{5}$ ), artificial neural networks (e.g. Lamedica et al. ${ }^{6}$ ), spectral methods (e.g. Laing and Smith ${ }^{7}$ ) and seasonal ARIMA models (e.g. Laing and Smith $^{7}$; Darbellay and Slama ${ }^{8}$ ). The most noticeable development in demand forecasting over the last decade has been the increasing interest shown by researchers and practitioners in artificial neural networks (see Hippert et al. ${ }^{9}$ ). Although there is obvious appeal to using this 
modelling approach to find the non-linear relationship between demand and weather variables, its appeal for univariate modelling is far less clear. The one short-term forecasting method that has remained popular over the years, and appears in many papers as a benchmark approach, is multiplicative seasonal ARIMA modelling.

The multiplicative seasonal ARIMA model, for a series, $X_{t}$, with just one seasonal pattern can be written as

$$
\phi_{p}(L) \Phi_{P}\left(L^{s}\right) \nabla^{d} \nabla_{s}^{D} X_{t}=\theta_{q}(L) \Theta_{Q}\left(L^{s}\right) \varepsilon_{t}
$$

where $L$ is the lag operator, $s$ is the number of periods in a seasonal cycle, $\nabla$ is the difference operator, $(1-L), \nabla_{s}$ is the seasonal difference operator, $\left(1-L^{S}\right), d$ and $D$ are the orders of differencing, $\varepsilon_{t}$ is a white noise error term, and $\phi_{p}, \Phi_{P}, \theta_{q}$ and $\Theta_{Q}$ are polynomial functions of orders $p, P, q$ and $Q$, respectively. The model is often expressed as $\operatorname{ARIMA}(p, d, q) \times(P, D, Q)_{s}$. It is multiplicative in the sense that the polynomial functions of $L$ and $L^{S}$ are multiplied on each side of the equation to give a rich function of the lag operator. Box et al. ${ }^{10}$ (p 333) comment that the model can be extended for the case of multiple seasonalities. The multiplicative double seasonal ARIMA model can be written as

$$
\phi_{p}(L) \Phi_{P_{1}}\left(L^{s_{1}}\right) \Omega_{P_{2}}\left(L^{s_{2}}\right) \nabla^{d} \nabla_{s_{1}}^{D_{1}} \nabla_{s_{2}}^{D_{2}} X_{t}=\theta_{q}(L) \Theta_{Q_{1}}\left(L^{s_{1}}\right) \Psi_{Q_{2}}\left(L^{s_{2}}\right) \varepsilon_{t}
$$

where $s_{1}$ and $s_{2}$ are the number of periods in the different seasonal cycles, and $\Omega_{P_{2}}$ and $\Psi_{Q_{2}}$ are polynomial functions of orders $P_{2}$ and $Q_{2}$, respectively. This model can be expressed as $\operatorname{ARIMA}(p, d, q) \times\left(P_{1}, D_{1}, Q_{1}\right)_{s_{1}} \times\left(P_{2}, D_{2}, Q_{2}\right)_{s_{2}}$. Applying the model to half-hourly electricity demand, Laing and Smith ${ }^{7}$ set $s_{1}=48$ to model the within-day seasonal cycle of 48 half-hours, and $s_{2}=336$ to model the within-week cycle of 336 half-hours. The forecasts from ARIMA models of this type are currently used at National Grid. In an application to hourly demand in the Czech Republic, Darbellay and Slama ${ }^{8}$ set $s_{1}=24$ to model the within-day seasonal cycle, and $s_{2}=168$ to model the within-week cycle. 
The multiplicative seasonal ARIMA model can easily be extended to take care of three or more seasonalities by the introduction of additional polynomial functions of the lag operator and additional difference operators in expression (1). Therefore, the annual seasonal pattern in electricity demand could also be modelled. However, it is usual to assume that it is not significant in the context of lead times up to a day-ahead ${ }^{7}$.

In this section, we have shown how the multiplicative double seasonal ARIMA model is a straightforward extension of the standard multiplicative seasonal model. Motivated by this, and by the fact that exponential smoothing has been a competitive alternative to ARIMA models with a variety of different types of data ${ }^{11}$, in the next section, we adapt the standard Holt-Winters method for application to series with two seasonalities.

\section{Double Seasonal Holt-Winters Exponential Smoothing}

\section{Standard Holt-Winters}

The standard Holt-Winters method was introduced by Winters ${ }^{12}$ and is suitable for series with one seasonal pattern. The multiplicative seasonality version of the method is presented in expressions (2)-(5). It assumes an additive trend and estimates the local slope, $T_{t}$, by smoothing successive differences, $\left(S_{t}-S_{t-1}\right)$, of the local level, $S_{t}$. The local s-period seasonal index, $I_{t}$, is estimated by smoothing the ratio of observed value, $X_{t}$, to local level, $S_{t}$.

$$
\begin{array}{ll}
\text { Level } & S_{t}=\alpha\left(X_{t} / I_{t-s}\right)+(1-\alpha)\left(S_{t-1}+T_{t-1}\right) \\
\text { Trend } & T_{t}=\gamma\left(S_{t}-S_{t-1}\right)+(1-\gamma) T_{t-1} \\
\text { Seasonality } & I_{t}=\delta\left(X_{t} / S_{t}\right)+(1-\delta) I_{t-s} \\
& \hat{X}_{t}(k)=\left(S_{t}+k T_{t}\right) I_{t-s+k}
\end{array}
$$

where $\alpha, \gamma$ and $\delta$ are smoothing parameters, and $\hat{X}_{t}(k)$ is the $k$-step-ahead forecast. The seasonality is multiplicative in the sense that the underlying level of the series is multiplied by the seasonal index. Holt-Winters for additive seasonality is an alternative formulation, which involves the addition of seasonal factors to the underlying trend. The multiplicative version is appropriate if the magnitude of the seasonal variation increases with an increase in 
the mean level of the series, while the additive version should be used if the seasonal effect does not depend on the current mean level. The multiplicative version is much more widely used and so for simplicity, in this paper, we provide only the multiplicative formulation.

It is worth noting that the use of the word "multiplicative" in the context of seasonal ARIMA models is quite different to its use in Holt-Winters exponential smoothing. By contrast with Holt-Winters for multiplicative seasonality, the seasonal effect for multiplicative seasonal ARIMA models does not depend on the mean level of the series. There is no equivalence between Holt-Winters for multiplicative seasonality and multiplicative seasonal ARIMA models. This point is, perhaps, emphasised by the fact that, although there is an ARIMA model for which Holt-Winters for additive seasonality is optimal $^{13}$, there is no ARIMA model for which Holt-Winters for multiplicative seasonality is optimal $^{14}$.

\section{Double Seasonal Holt-Winters}

Although standard Holt-Winters is widely used for forecasting seasonal time series, the method is only able to accommodate one seasonal pattern. A formulation that can accommodate more than one seasonal pattern has not been considered in the exponential smoothing literature. This is evident from the recent taxonomies of Hyndman et al. ${ }^{15}$ and Taylor $^{16}$. The Holt-Winters method for double multiplicative seasonality is given in expression (6)-(10). The method is suitable when there are two seasonal patterns in the time series. The formulation involves separate seasonal indices, $D_{t}$ and $W_{t}$, for the two seasonalities. The local $s_{1}$-period seasonal index, $D_{t}$, is estimated by smoothing the ratio of observed value, $X_{t}$, to the product of the local level, $S_{t}$, and local $s_{2}$-period seasonal index, $W_{t-s_{2}}$. Similarly, the local $s_{2}$-period seasonal index, $W_{t}$, is estimated by smoothing the ratio of observed value, $X_{t}$, to the product of the local level, $S_{t}$, and local $s_{1}$-period seasonal index, $D_{t-S_{1}}$. 
Level

$$
S_{t}=\alpha\left(X_{t} /\left(D_{t-s_{1}} W_{t-s_{2}}\right)\right)+(1-\alpha)\left(S_{t-1}+T_{t-1}\right)
$$

Trend

$T_{t}=\gamma\left(S_{t}-S_{t-1}\right)+(1-\gamma) T_{t-1}$

Seasonality 1

$$
D_{t}=\delta\left(X_{t} /\left(S_{t} W_{t-S_{2}}\right)\right)+(1-\delta) D_{t-S_{1}}
$$

Seasonality 2

$$
\begin{aligned}
& W_{t}=\omega\left(X_{t} /\left(S_{t} D_{t-s_{1}}\right)\right)+(1-\omega) W_{t-s_{2}} \\
& \hat{X}_{t}(k)=\left(S_{t}+k T_{t}\right) D_{t-s_{1}+k} W_{t-s_{2}+k}
\end{aligned}
$$

where $\alpha, \gamma, \delta$ and $\omega$ are smoothing parameters. Applying the method to a series of half-hourly demand, one would set $s_{1}=48$ and $s_{2}=336$, as in the multiplicative double seasonal ARIMA model of Laing and Smith ${ }^{7} . D_{t}$ and $W_{t}$ would then represent the within-day and within-week seasonalities, respectively. A double additive seasonality method can be developed in a similar way from the standard Holt-Winters method for additive seasonality. The formulation in expressions (6)-(10) can easily be extended for three or more seasonal patterns by introducing an extra seasonal index and smoothing equation for each additional seasonality.

\section{Empirical Comparison of Methods}

We carried out empirical analysis in order to address two main issues. Firstly, we wished to investigate whether the new double seasonal Holt-Winters method offers an improvement on the standard Holt-Winters method in terms of forecast accuracy. Secondly, we wanted to compare forecasting performance of the new formulation with a well-specified multiplicative double seasonal ARIMA model.

The data used was 12 weeks of half-hourly electricity demand in England and Wales from Monday 5 June 2000 to Sunday 27 August 2000. It is shown in Figure 2. We used the first 8 weeks of data to estimate method parameters and the remaining 4 weeks to evaluate post-sample forecasting performance. This amounts to 2,688 half-hourly observations for estimation and 1,344 for evaluation. To simplify our comparison of methods, we chose a period that did not contain any 'special' days, such as national holidays. Demand on these days is so very unlike the rest of the year that online univariate methods are generally unable to produce reasonable forecasts. In practice, interactive facilities tend to be used for special 
days, which allow operator experience to supplement or override the system offline. If a forecasting method is unable to tolerate gaps in the historical series, the special days can be smoothed over, leaving the natural periodicities of the data intact ${ }^{7}$.

$* * * * *$ Figure $2 * * * * *$

\section{Multiplicative Double Seasonal ARIMA}

The process of model identification is impractical in an online demand forecasting system, and so the model is chosen offline. We used the Box-Jenkins modelling methodology to identify the most suitable ARIMA model based on the 2,688 observations in the estimation sample. The autocorrelation function and partial autocorrelation function were used to select the order of the model, which was then estimated by maximum likelihood. The residuals were inspected for any remaining autocorrelation. Laing and Smith $^{7}$ explain that, in the multiplicative double seasonal ARIMA formulation in expression (1), polynomials of order greater than two are rarely necessary when fitting a model to half-hourly data for England and Wales. In view of this, we considered polynomials up to order two, but we also checked the autocorrelation function of the residuals for any remaining higher order autocorrelation. We compared the Schwartz Bayesian Criterion (SBC) for an extensive range of different ARIMA models. We investigated differencing and a logarithmic transformation for demand but found neither to improve the SBC. The model with lowest SBC and satisfactory residuals was the following $\operatorname{ARIMA}(2,0,0) \times(2,0,2)_{48} \times(2,0,2)_{336}$ model, which we shall refer to as the Double Seasonal ARIMA model:

$$
\begin{aligned}
\left(1-1.02 L+0.08 L^{2}\right)\left(1-0.32 L^{48}-0.31 L^{96}\right)\left(1-0.52 L^{336}-\right. & \left.0.44 L^{672}\right)\left(X_{t}-21,625\right) \\
& =\left(1-0.16 L^{96}\right)\left(1-0.15 L^{336}-0.19 L^{672}\right) \varepsilon_{t}
\end{aligned}
$$




\section{Holt-Winters Exponential Smoothing}

We produced forecasts using the following three Holt-Winters methods:

Holt-Winters for Within-Day Seasonality - This is standard Holt-Winters for multiplicative seasonality, given in expressions (2)-(5), using only a 48-period seasonal cycle.

Holt-Winters for Within-Week Seasonality - This is standard Holt-Winters for multiplicative seasonality, given in expressions (2)-(5), using only a 336-period seasonal cycle.

Double Seasonal Holt-Winters - This is the new Holt-Winters for double multiplicative seasonality, given in expressions (6)-(10), using both a 48-period cycle for the within-day seasonality and a 336-period seasonal cycle for the within-week seasonality.

Williams and Miller ${ }^{17}$ use simple averages of the first few data observations to calculate initial smoothed values for the level, trend and seasonal components in the standard Holt-Winters method. We implemented their procedure for Holt-Winters for Within-Day Seasonality and Holt-Winters for Within-Week Seasonality. We adapted the procedure for Double Seasonal Holt-Winters. The initial trend, $T_{0}$, was chosen as the average of (1) $1 / 336$ of the difference between the mean of the first 336 and second 336 observations, and (2) the average of the first differences for the first 336 observations. The initial level, $S_{0}$, was chosen as the mean of the first 672 observations minus 336.5 times the initial trend. The initial values for the within-day seasonal index, $D_{t}$, were set as the average of the ratios of actual observation to 48-point centred moving average, taken from the corresponding half-hour period in each of the first seven days of the time series. The initial values for the within-week seasonal index, $W_{t}$, were set as the average of the ratios of actual observation to 336-point centred moving average, taken from the corresponding half-hour period on the same day of the week in each of the first two weeks of the demand series, divided by the corresponding initial value of the smoothed within-day seasonal index, $D_{t}$.

We derived parameter values by the common procedure of minimising the sum of squared 1-step-ahead forecast errors using a non-linear optimisation routine. The estimated 
parameters are shown in Table 1. The zero values for $\gamma$ in Holt-Winters for Within-Week Seasonality and Double Seasonal Holt-Winters were accompanied by a constant small value for the smoothed trend, $T_{t}$. This seems reasonable since variation in the 8-week estimation period is dominated by seasonality. The high value for $\gamma$ in Holt-Winters for Within-Day Seasonality was accompanied by highly varying, and quite sizeable, values for the smoothed trend. Since the method is unable to pick up the weekly seasonality in the data, it has incorporated this variability in its estimate of the trend.

$* * * * *$ Table 1

Figure 3 compares the post-sample forecasting accuracy of the three Holt-Winters methods and the ARIMA model for lead times up to a day-ahead. The figure shows the mean absolute percentage error (MAPE), which is the most widely used error summary measure in electricity demand forecasting. Following the recommendation of Hippert et al. ${ }^{9}$, we also calculated the mean absolute error, root mean square error and root mean square percentage error, but we do not report these results here because the relative performances of the methods for these measures were very similar to those for the MAPE. The results for HoltWinters for Within-Day Seasonality were so poor that it was impractical to plot the MAPE values beyond 2-steps-ahead ahead on the same graph as the MAPE values for the other methods. This is due to the method failing to accommodate within-week seasonality. This might have been anticipated from Figure 1, which shows how very different demand on Saturdays and Sundays is from demand on weekdays. Holt-Winters for Within-Week Seasonality is far more competitive, suggesting that the within-week seasonality accounts for a large proportion of the variation in the data. However, Double Seasonal Holt-Winters outperforms Holt-Winters for Within-Week Seasonality for 38 of the 48 lead times, indicating that there is benefit in using a method that is able to pick up both seasonalities. Beyond 12 hours-ahead, the accuracy of these two methods tends to improve with the lead time. This is due to the within-day seasonality, and it implies that a forecast for 12 hours ahead would be 
better made from a forecast origin 12 hours prior to the current period. In his analysis of online methods, Smith ${ }^{18}$ also concludes that the choice of forecast origin should depend on the forecast horizon. Comparing the two double seasonal methods, we see that Double Seasonal ARIMA outperforms Double Seasonal Holt-Winters for all but the last 5 lead times.

Figure 3

\section{Adjusting for Error Autocorrelation in the Holt-Winters Methods}

Inspection of the 1-step-ahead errors, in the estimation sample of 2,688 periods, revealed sizeable first-order autocorrelation for all three Holt-Winters methods, indicating that the forecasts were suboptimal. Gardner ${ }^{19}$ reports how the forecasts from exponential smoothing methods can sometimes be improved by using a simple adjustment, initially proposed by Reid $^{20}$ and Gilchrist ${ }^{21}$ (pp 202-203). The adjustment involves an AR(1) model, $e_{t}$ $=\lambda e_{t-1}+\xi_{t}$, being fitted to the 1 -step-ahead errors, $e_{t}$. The $k$-step-ahead forecasts from forecast origin $\tau$ are then modified by adding the term $\lambda^{k} e_{\tau}$. Chatfield ${ }^{22}$ found that the modification resulted in improvements in accuracy when applied to the autocorrelated errors from Holt-Winters for multiplicative seasonality. Using just the estimation sample, we fitted AR(1) models to the residuals from each of the three Holt-Winters methods described in the previous section. This led to improved post-sample results for all three methods at the very early lead times.

Estimating the parameters of a Holt-Winters method and then fitting a model to the residuals is a two-stage estimation approach. Chatfield ${ }^{22}$ suggests that it may be more efficient to estimate all of the parameters for a method in a single stage. We did this for each of the three Holt-Winters methods by minimising the sum of squared 1-step-ahead errors from the estimation sample. This led to far greater improvements in post-sample accuracy than were found using the two-stage estimation approach. Before presenting the post-sample MAPE results, let us first consider the estimated parameters resulting from the single-stage 
estimation approach. These are shown in Table 2. The parameters are noticeably different to those shown in Table 1 for the same methods without residual autocorrelation adjustment. The introduction of the AR(1) model for the residuals has caused a sizeable reduction in the smoothing parameter for the level in Holt-Winters for Within-Week Seasonality and Double Seasonal Holt-Winters. It would seem that the introduction of the model for the residuals has, to a large degree, replaced the smoothing equation for the level. Incidentally, we did not fit a model to the residuals of the Double Seasonal ARIMA model because it was estimated after careful diagnostic evaluation, and so there was no autocorrelation in its residuals.

\section{***** Table 2 *****}

Figure 4 shows the post-sample forecasting performance for the three Holt-Winters methods with residual autocorrelation adjustment. The MAPE results for Double Seasonal ARIMA, which were plotted in Figure 3, are also shown in Figure 4. The new results for all three Holt-Winters methods have improved substantially from Figure 3. The relative performance of the three methods has not changed, but Double Seasonal Holt-Winters is now the best of the three for all 48 lead times. Interestingly, the method now also outperforms Double Seasonal ARIMA for all the lead times. Beyond 12-periods-ahead, the ARIMA model is also outperformed by Holt-Winters for Within-Week Seasonality.

\section{$* * * * *$ Figure $4 * * * * *$}

Intuitively, it is not surprising that, for the electricity demand data, the new double seasonal Holt-Winters method was more accurate than the two implementations of the standard Holt-Winters method. Application of the standard method was relatively naïve because it is unable to accommodate more than one seasonality. Nevertheless, it is pleasing to find that the empirical results support intuition. It is less clear why the new method with residual autocorrelation adjustment outperforms the ARIMA model. It cannot simply be due to the multiplicative nature of the double seasonal Holt-Winters method because an additive version of the method performed similarly. A possible explanation is provided by the 
comments of Chatfield ${ }^{23}$ and Chatfield and $\mathrm{Yar}^{24}$ in their consideration of the choice between ARIMA modelling and exponential smoothing. They explain that ARIMA modelling is worth considering if the series is dominated by short-term correlation but not when it is dominated by trend and seasonal variation. Since electricity demand, recorded at half-hourly or hourly intervals, is dominated by seasonal variation, it follows that Holt-Winters formulations should perform well in comparison with ARIMA models.

\section{Summary and Conclusions}

Online short-term electricity demand forecasting requires a robust, univariate procedure. Inspection of a time series of half-hourly demand reveals a within-day seasonality and a within-week seasonality. A popular approach is to use a multiplicative double seasonal ARIMA model. The robustness of exponential smoothing methods suggests that HoltWinters would be a reasonable candidate for online short-term demand forecasting. However, the method is only able to accommodate one seasonal pattern. In this paper, we have shown how the method can be adapted for time series with two seasonalities. This involves the introduction of an additional seasonal index and an extra smoothing equation for this new seasonal index.

Using a series of half-hourly electricity demand, the new formulation outperformed standard Holt-Winters for forecast lead times from a half-hour-ahead to a day-ahead. The Holt-Winters methods were improved by the inclusion of an AR(1) model for the residuals. The best results were achieved by estimating the AR(1) model parameter in the same estimation procedure as the exponential smoothing parameters. The resulting forecasts for the new double seasonal Holt-Winters method outperformed those from standard Holt-Winters and also those from a well-specified multiplicative double seasonal ARIMA model. We, therefore, conclude that there is strong potential for the use of the new double seasonal HoltWinters formulation in online short-term electricity demand forecasting. However, rather 
than recommending the new method in preference to all others, we feel that a more useful approach would be to use several different methods. Smith ${ }^{18}$ discusses combining online electricity demand forecasts from different methods with weights varying according to the particular period of the week and the forecast origin. Taylor and Majithia ${ }^{25}$ describe how $^{2}$ smooth transition combining methods can used to enable a smooth form of switching between different demand forecasting methods.

\section{Acknowledgements}

We are grateful to Shanti Majithia, Chris Rogers and Sal Sabbagh of National Grid for supplying data and information regarding the company's online demand forecasting. We are also grateful for the useful comments of the anonymous referees.

\section{References}

1 Taylor JW and Buizza R (2003). Using weather ensemble predictions in electricity demand forecasting. International Journal of Forecasting 19: 57-70.

2 Taylor JW and Buizza R (2002). Neural Network Load Forecasting with Weather Ensemble Predictions. IEEE Transactions on Power Systems 17: 626-632.

3 Bunn DW (1982). Short-term forecasting: A review of procedures in the electricity supply industry. Journal of the Operational Research Society 33: 533-545.

4 Infield DG and Hill DC (1998). Optimal smoothing for trend removal in short-term electricity demand forecasting. IEEE Transactions Power Systems 13: 1115-1120.

5 Christiaanse WR (1971). Short-term load forecasting using general exponential smoothing. IEEE Trans. on Power Apparatus and Systems Pas-90: 900-902.

6 Lamedica R, Prudenzi A, Sforna M, Caciotta M and Cencelli VO (1996). A neural network based technique for short-term forecasting of anomalous load periods. IEEE Transactions Power Systems 11: 1749-1756.

$7 \quad$ Laing WD and Smith DGC (1987). A comparison of time series forecasting methods for predicting the CEGB demand. Proceedings of the Ninth Power Systems Computation Conference.

8 Darbellay GA and Slama M (2000). Forecasting the short-term demand for electricity - Do neural networks stand a better chance? International Journal of Forecasting 16: 71-83. 
9 Hippert HS, Pedreira CE and Souza RC (2001). Neural networks for short-term load forecasting: A review and evaluation. IEEE Transactions on Power Systems 16: 44-55.

10 Box GEP, Jenkins GM and Reinsel GC (1994). Time Series Analysis: Forecasting and Control, third edition. Englewod Cliffs, Prentice Hall: New Jersey, p 333.

11 Makridakis S, Chatfield C, Hibon M, Lawrence M, Mills T, Ord K and Simmons LF (1993). The M2-Competition: A real-time judgementally based forecasting study. International Journal of Forecasting 9: 5-22.

12 Winters PR (1960). Forecasting sales by exponentially weighted moving averages. Management Science 6: 324-342.

13 McKenzie E (1976). A comparison of some standard seasonal forecasting systems. The Statistician 25: 3-14.

14 Abraham B and Ledolter J (1986). Forecast functions implied by autoregressive integrated moving average and other related forecast procedures. International Statistical Review 54: 51-66.

15 Hyndman RJ, Koehler AB, Snyder RD and Grose S (2002). A state space framework for automatic forecasting using exponential smoothing methods. International Journal of Forecasting 18: 439-454.

16 Taylor JW (2003). Damped multiplicative trend exponential smoothing. International Journal of Forecasting forthcoming.

17 Williams DW and Miller D (1999). Level-adjusted exponential smoothing for modeling planned discontinuities. International Journal of Forecasting 15: 273-289.

18 Smith DGC (1989). Combination of forecasts in electricity demand prediction. Journal of Forecasting 8: 349-356.

19 Gardner ES Jr. (1985). Exponential smoothing: The state of the art. Journal of Forecasting 4: 1-28.

20 Reid DJ (1975). A review of short-term projection techniques. In: Practical Aspects of Forecasting, Gordon HA (ed.). Operational Research Society, London, pp 8-25.

21 Gilchrist W (1976). Statistical Forecasting. Wiley: Chichester.

22 Chatfield C (1978). The Holt-Winters forecasting procedure. Applied Statistics 27: 264-279.

23 Chatfield C (1985). Comments on 'Exponential smoothing: The state of the art' by Gardner ES Jr. Journal of Forecasting 4: 30.

24 Chatfield C and Yar M (1988). Holt-Winters forecasting: Some practical issues. The Statistician 37: 129-140. 
25 Taylor JW and Majithia S (2000). Using combined forecasts with changing weights for electricity demand profiling. Journal of the Operational Research Society 51: 7282. 


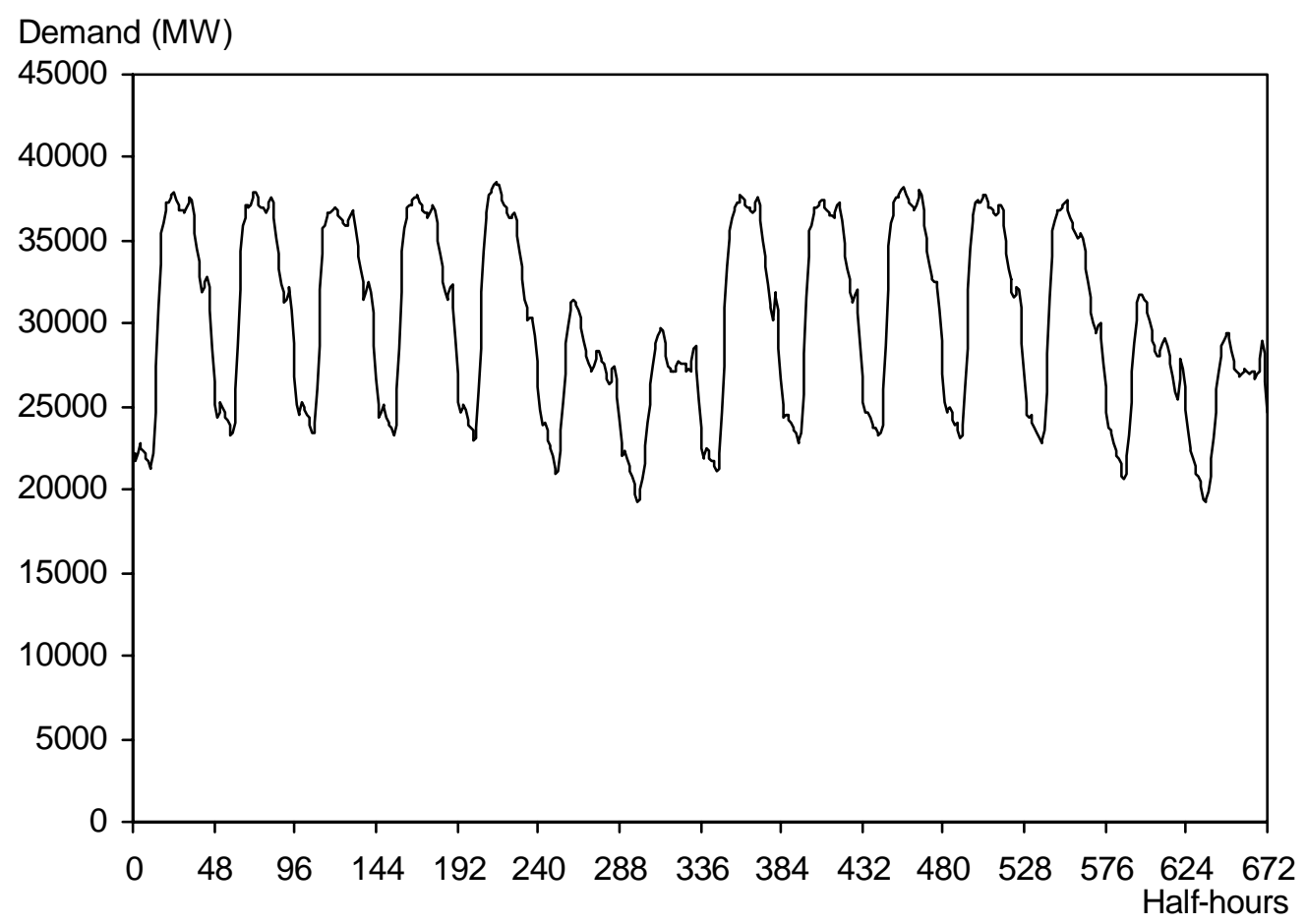

Figure 1 Half-hourly electricity demand in England and Wales from Monday 5 June 2000 to Sunday 18 June 2000. 


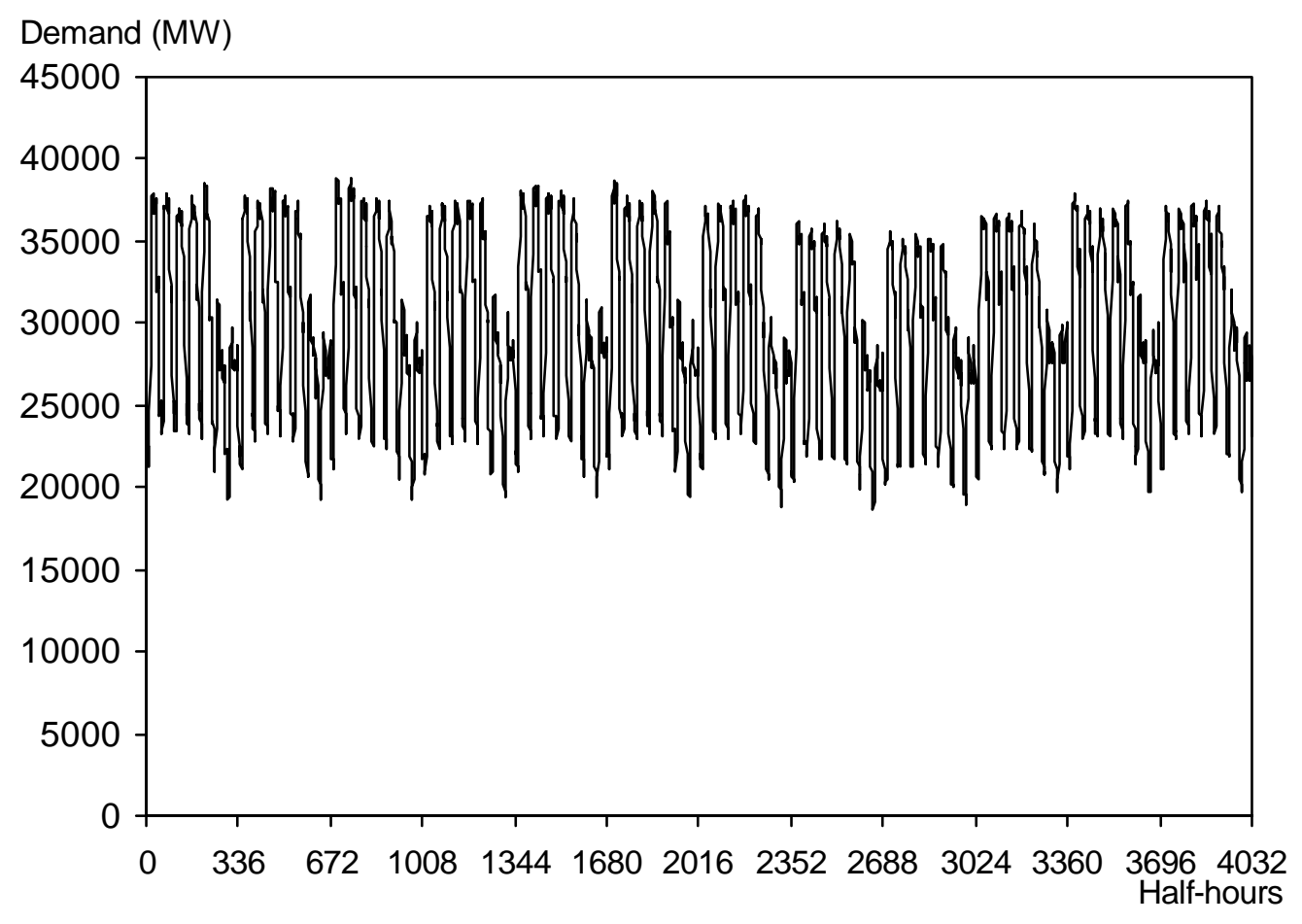

Figure 2 Half-hourly electricity demand in England and Wales from Monday 5 June 2000 to Sunday 27 August 2000. 


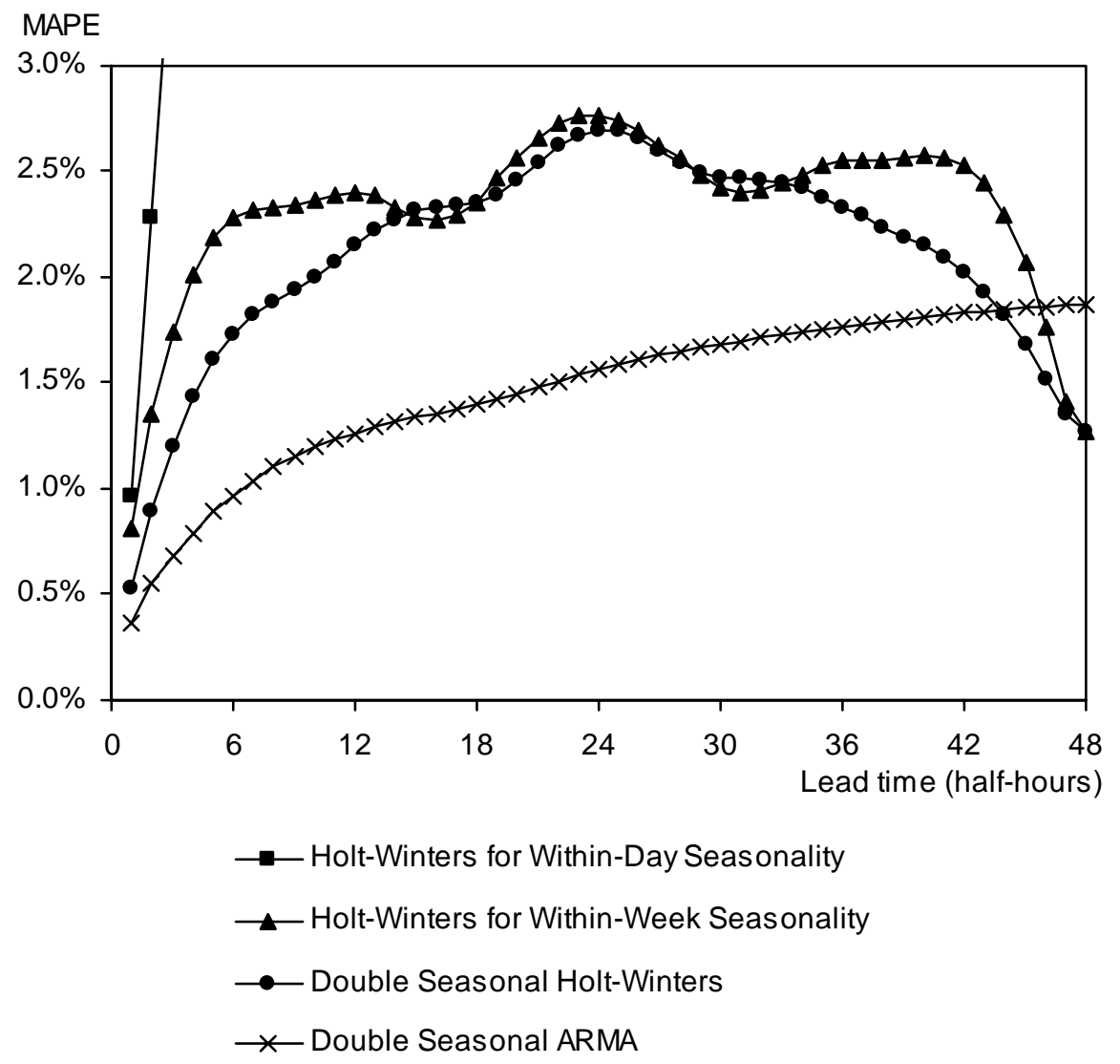

Figure 3 Comparison of MAPE results for the 4-week post-sample. No adjustment for autocorrelation in the residuals of the Holt-Winters methods. 


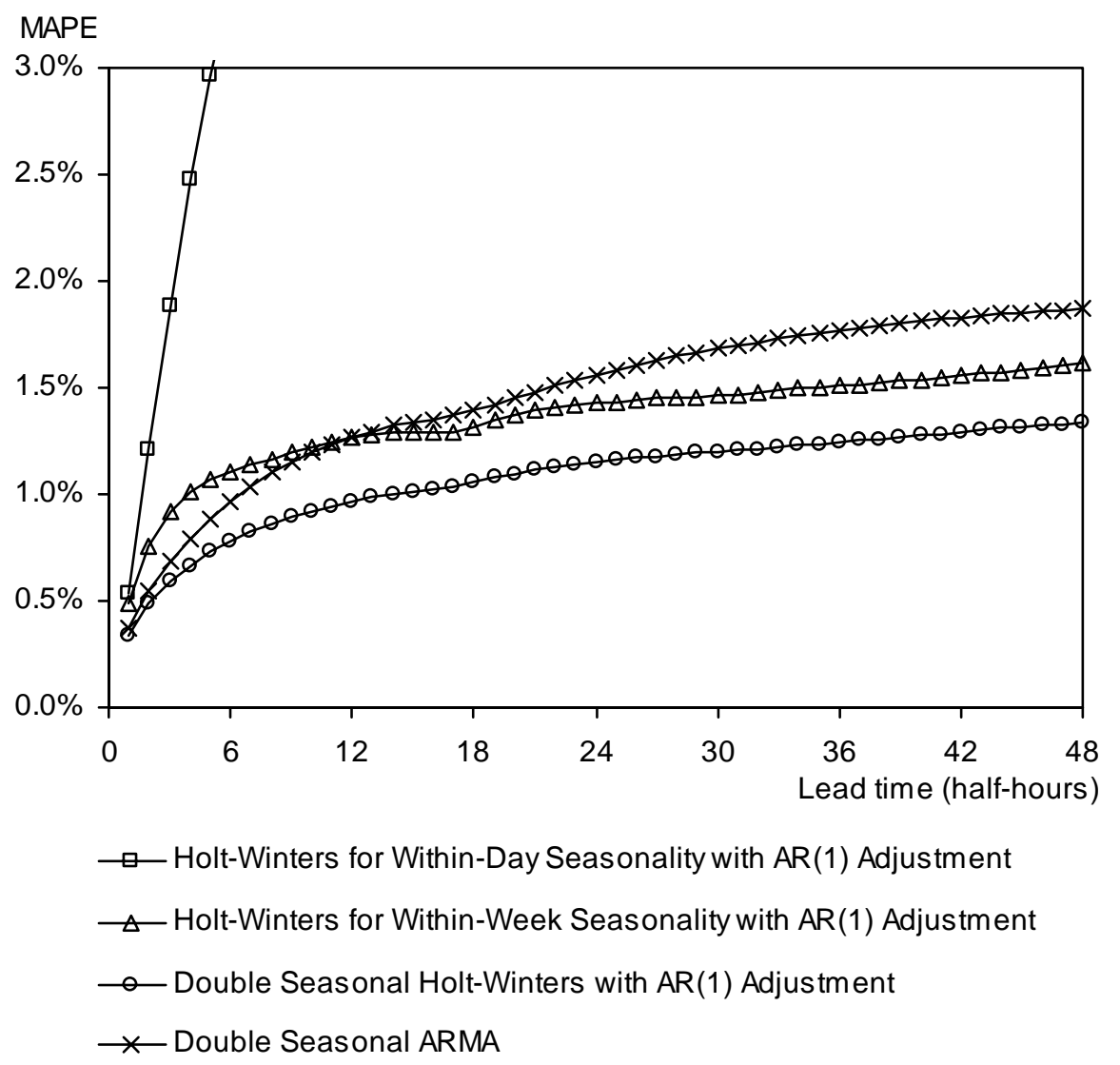

Figure 4 Comparison of MAPE results for the 4-week post-sample period. The Holt-Winters methods included an AR(1) model for the residuals. 
Table 1 Holt-Winters parameters calculated from the 8-week estimation sample. No adjustment for autocorrelation in the residuals of the methods.

\begin{tabular}{l|cccc} 
& $\begin{array}{c}\text { Level } \\
\alpha\end{array}$ & $\begin{array}{c}\text { Trend } \\
\gamma\end{array}$ & $\begin{array}{c}\text { Within-day } \\
\text { seasonality } \delta\end{array}$ & $\begin{array}{c}\text { Within-week } \\
\text { seasonality } \omega\end{array}$ \\
\hline Holt-Winters for Within-Day Seasonality & 0.98 & 0.90 & 1.00 & - \\
Holt-Winters for Within-Week Seasonality & 0.82 & 0.00 & - & 1.00 \\
Double Seasonal Holt-Winters & 0.90 & 0.00 & 1.00 & 1.00
\end{tabular}

Table 2 Holt-Winters parameters calculated from the 8-week estimation sample. The methods included an AR(1) model for the residuals.

\begin{tabular}{l|ccccc} 
& $\begin{array}{c}\text { Level } \\
\alpha\end{array}$ & $\begin{array}{c}\text { Trend } \\
\gamma\end{array}$ & $\begin{array}{c}\text { Within-day } \\
\text { seasonality } \delta\end{array}$ & $\begin{array}{c}\text { Within-week } \\
\text { seasonality } \omega\end{array}$ & $\begin{array}{c}\text { AR } \\
\lambda\end{array}$ \\
\hline Holt-Winters for Within-Day Seasonality & 0.92 & 0.00 & 1.00 & - & 0.71 \\
Holt-Winters for Within-Week Seasonality & 0.02 & 0.00 & - & 0.41 & 0.91 \\
Double Seasonal Holt-Winters & 0.01 & 0.00 & 0.21 & 0.24 & 0.92
\end{tabular}

